CERN-PH-TH/2007-001

$\mathrm{UCLA} / 07 / \mathrm{TEP} / 1$

\title{
Critical points of the Black-Hole potential for homogeneous special geometries
}

\author{
R. D’Auria ${ }^{\star}$, S. Ferrara ${ }^{\dagger}$ and M. Trigiante ${ }^{\star}$ \\ ^ Dipartimento di Fisica, Politecnico di Torino \\ C.so Duca degli Abruzzi, 24, I-10129 Torino, and \\ Istituto Nazionale di Fisica Nucleare, \\ Sezione di Torino, Italy \\ E-mail: riccardo.dauria@polito.it, mario.trigiante@polito.it \\ $\dagger$ CERN, Physics Department, CH 1211 Geneva 23, Switzerland \\ and \\ INFN, Laboratori Nazionali di Frascati, Italy \\ and \\ Department of Physics \& Astronomy, University of California, Los Angeles, CA, USA \\ E-mail: Sergio.Ferrara@cern.ch
}

\begin{abstract}
We extend the analysis of $\mathrm{N}=2$ extremal Black-Hole attractor equations to the case of special geometries based on homogeneous coset spaces. For non-BPS critical points (with non vanishing central charge) the (Bekenstein-Hawking) entropy formula is the same as for symmetric spaces, namely four times the square of the central charge evaluated at the critical point. For non homogeneous geometries the deviation from this formula is given in terms of geometrical data of special geometry in presence of a background symplectic charge vector.
\end{abstract}




\section{Introduction}

A remarkable feature of extremal black-holes in supergravity is the attractor mechanism which is at work for both BPS [1] and non-BPS [2, 3, 4, 5, 6, 7, 8, solutions: Starting from generic boundary values at radial infinity, the scalar fields coupled to the black-hole evolve towards fixed values at the horizon which are functions of the quantized electric and magnetic charges only. These fixed values are determined by the minimum of a certain effective potential $V_{B H}$ which depends on the scalar fields and on the charges. Extremal black holes in four dimensions feature a near-horizon geometry of the form $A d S_{2} \times S^{2}$. The area of the horizon, which is expressed in terms of the radius of $S^{2}$ in the throat of the solution, is given by the value of $V_{B H}$ at its minimum and is related via the Bekenstein-Hawking (B-H) formula, to the entropy of the black hole

$$
S=\frac{A_{H}}{4}=\pi V_{B H \mid e x t r .} .
$$

Although the attractor mechanism was shown to be at work for BPS solutions also in the presence of higher derivative corrections [9], we shall restrict our analysis to second order derivative supergravity action. Extremal black holes play an important role in the microscopic/statistical interpretation of the B-H expression for the entropy possibly corrected by higher derivative contributions [10, 11, 12, 13, 14, 15, 16] (for a recent review on black holes see [17]).

Recently the critical points of the black-hole potential $V_{B H}$ in $N=2, D=4$ supergravity coupled to vector multiplets have been studied and classified for all homogeneous symmetric special Kähler geometries [18. It is the goal of the present paper to extend the analysis to homogeneous non-symmetric spaces whose general classification was given and discussed in [19, 20, 21]. The nature of these spaces is quite interesting from a physical point of view since some of them naturally appear in brane dynamics, when the brane and bulk degrees of freedom are unified in the four dimensional effective supergravity. Homogeneous special geometries can be classified by coset spaces $\mathcal{M}=G / H$ where

$$
\begin{aligned}
G & =\left[\mathrm{SO}(1,1)_{0} \times \mathrm{SO}(2,2+q)\right] \ltimes \mathcal{N}, \\
H & =\mathrm{SO}(2) \times \mathrm{SO}(2+q),
\end{aligned}
$$

and $\mathcal{N}$ is a subgroup generated by a nilpotent graded subalgebra $\mathbb{N}$ of the isometry algebra:

$$
\begin{aligned}
\mathcal{N} & =\exp (\mathbb{N}), \\
\mathbb{N} & =W^{(+1)} \oplus W^{(+2)},
\end{aligned}
$$

where the superscripts refer to the $\mathrm{SO}(1,1)_{0}$-grading. The generators $T_{\alpha}$ of $W^{(+1)}$ transform in a real (in general reducible) $d$-dimensional spinorial representation of $\mathrm{SO}(2, q+2)$ while $W^{(+2)}$ is one-dimensional and its the generator $T_{\bullet}$ is an $\mathrm{SO}(2, q+2)$-singlet. The algebraic structure of $\mathbb{N}$ is defined by the following commutation relations:

$$
\left[T_{\alpha}, T_{\beta}\right]=2 C_{\alpha \beta} T_{\bullet} ;\left[T_{\alpha}, T_{\bullet}\right]=0,
$$


$C_{\alpha \beta}$ being the antisymmetric real matrix to be identified with the charge conjugation matrix of the spinorial representation defined by $T_{\alpha}$ (see appendix $\mathrm{B}$ ). The number of complex coordinate of the manifold is

$$
n=\operatorname{dim}_{\mathbb{C}} \mathcal{M}=3+q+\frac{d}{2} .
$$

Denoting by $T_{\Lambda \Sigma}=-T_{\Sigma \Lambda}(\Lambda, \Sigma=0,1, \ldots, q+3)$ the $\mathrm{SO}(2,2+q)$ generators and by $h_{0}$ the $\mathrm{SO}(1,1)_{0}$ generator, the following commutation relations hold:

$$
\begin{aligned}
{\left[T_{\Lambda \Sigma}, T_{\Gamma \Delta}\right] } & =\frac{1}{2}\left(\eta_{\Lambda \Delta} T_{\Sigma \Gamma}+\eta_{\Sigma \Gamma} T_{\Lambda \Delta}-\eta_{\Lambda \Gamma} T_{\Sigma \Delta}-\eta_{\Sigma \Delta} T_{\Lambda \Gamma}\right) \\
{\left[T_{\Lambda \Sigma}, T_{\alpha}\right] } & =-\frac{1}{4}\left(\Gamma_{\Lambda \Sigma}\right)_{\alpha}{ }^{\beta} T_{\beta} ;\left[h_{0}, T_{\alpha}\right]=T_{\alpha} ;\left[h_{0}, T_{\bullet}\right]=2 T_{\bullet}
\end{aligned}
$$

all the other commutators between the same generators being zero. The $d / 2$ complex coordinates parametrizing $T_{\alpha}$ define an $\mathrm{SO}(2,2+q)$ Clifford module. The compact isometries are $\mathrm{SO}(2) \times \mathrm{SO}(2+q) \times \mathcal{S}_{q}(P, \dot{P})$, and include the centralizer $\mathcal{S}_{q}(P, \dot{P})$ of the Clifford algebra representation [20], which is not contained inside $G$. In the table below we summarize the notations introduced in 20] for the classification of homogeneous special Kähler manifolds.

\begin{tabular}{|c|c|}
\hline $\mathcal{M}$ & $d$ \\
\hline$L(0, P, \dot{P})$ & $2(P+\dot{P})$ \\
\hline$L(q, P, \dot{P}), q=4 m>0$ & $4 D_{q}(P+\dot{P})$ \\
\hline$L(q, P), q>0, q \neq 4 m$ & $4 D_{q} P$ \\
\hline$L(-1, P)$ & $2 P$ \\
\hline$L(-2, P)$ & $2 P$ \\
\hline
\end{tabular}

where the values for $D_{q}$ are:

$$
D_{1}=1, D_{2}=2, D_{3}=D_{4}=4, D_{5}=D_{6}=D_{7}=D_{8}=8 \text {. }
$$

Certain homogeneous (non-symmetric) spaces listed in Table 1 naturally appear in brane dynamics, for instance in Type IIB compactification on $K 3 \times T^{2} / \mathbb{Z}_{2}$ orientifold in the presence of space-filling $D 3$ and $D 7$ branes $[22,23,24$. In this case the relevant space is $L(0, P, \dot{P})$, which is parametrized by the $S, T, U$ moduli of the bulk as well as the $P$ and $\dot{P}$ (which may be different from $P$ ) complex moduli describing the positions of the $D 3$ and $D 7$ branes along $T^{2}$ respectively. The model has the additional global symmetry $\mathcal{S}_{0}(P, \dot{P})=\mathrm{SO}(P) \times \mathrm{SO}(\dot{P})$ which is not part of the isometry group $G$, whose compact subgroup is $\mathrm{SO}(2) \times \mathrm{SO}(2)$ in this case. This state of affair is actually a particular case of a more general feature of $L(q, P, \dot{P})$ spaces for which the rigid symmetry $S_{q}(P, \dot{P})$ depends in general on $q$ as well as on the number of Clifford moduli.

The (rank three) symmetric spaces fall in the class $L(0, P)$ (which is the same as $L(q, 0)$ for $q=P)$ and $L(q, 1)$ with $q=1,2,4,8$ which correspond to the magic supergravities. The spaces $L(q, 0)$ are obtained by setting $T_{\alpha}=0$ and define the infinite series of manifolds of the form

$$
\mathcal{M}=\frac{\mathrm{SU}(1,1)}{\mathrm{U}(1)} \times \frac{\mathrm{SO}(2,2+q)}{\mathrm{SO}(2) \times \mathrm{SO}(q+2)} .
$$


In this context the STU model corresponds to $L(0,0)$ and is the smallest rank three symmetric space. In the magic supergravity cases the real dimension of the Clifford module is respectively given by: $d=4,8,16,32$, corresponding to the Freudenthal triple system of the Jordan algebras on $\mathbb{R}, \mathbb{C}, \mathbb{H}, \mathbb{O}$ respectively. For all the symmetric spaces the $H$-connection $\Omega^{(H)}$ actually coincides with the torsion-free Riemann connection $\omega$, while they differ in the nonsymmetric case. This will be explicitly shown in the next section.

The black-hole attractors for the $S, T, U$ model are clearly connected to the $N=8$ attractors studied in [3]. On the other hand the black hole attractors for all symmetric spaces were studied in [18]. There it was found that such attractors fall into three classes of orbits: One BPS and two non-BPS. The latter were further distinguished by the fact that the $N=2$ central charge $Z$ is non-vanishing in one case and vanishing in the other. It is the scope of the present paper to generalize this classification to the homogeneous spaces which are not symmetric.

We actually find that for the non-BPS orbit with $|Z| \neq 0$ the same rule as for the symmetric case applies, being

$$
V_{B H \mid \text { extr. }}=4|Z|_{\mid \text {extr. }}^{2} .
$$

The paper is organized as follows. In section 2 we give the special geometry for homogeneous spaces in rigid, namely tangent space, indices. We further compute the Riemann spin connection and the $\mathrm{H}$-connection for the cosets. In sections 3 and 4 we analyze the attractor equations for both $Z \neq 0$ and $Z=0$ orbits. While in the case $Z \neq 0$ the situation is similar to the symmetric case (in particular we get the same expression for the entropy in terms of the central charge), the classification is more involved in the $Z=0$ case, where many orbits seem to exist. Finally some useful mathematical tools for special geometry describing homogeneous spaces are collected in the appendices.

\section{Special geometry in rigid indices}

In the present section we shall explicitly construct the tensor quantities on a homogeneous special Kähler manifold, which will be relevant to our discussion in a rigid basis. In appendix A we review some basic facts and definitions about special geometry. The following discussion however relies only on the isometry properties of these spaces, outlined in the introduction, and will not make use of special Kähler identities.

We start from writing the connection and curvature in a real basis of the tangent space of the manifold and then introduce the rigid metric and complex structure. We shall choose as a basis for the tangent space of the symmetric submanifold $\mathrm{SO}(2,2+q) / \mathrm{SO}(2) \times \mathrm{SO}(q+2)$ the $2(2+q)$ non-compact generators $T_{a I}, a=0,1$ and $I=2, \ldots, q+3$, defined by the Cartan decomposition of the $\mathfrak{s o}(2,2+q)$ algebra. The corresponding basis of the tangent space of the whole homogeneous manifold will therefore consist of the following generators

$$
\left\{T_{A}\right\}=\left\{h_{0}, T_{a I}, T_{\alpha}, T_{\bullet}\right\} ; A=1, \ldots, 2 n
$$


The vielbeins dual to the isometry generators $\left\{T_{A}\right\}$ are $\left\{V^{A}\right\}=\left\{V^{0}, V^{a I}, V^{\alpha}, V^{\bullet}\right\}$ which, together with the $\mathrm{SO}(2) \times \mathrm{SO}(q+2)$-connection 1 -forms $\Omega^{(H) \lambda}=\left(\Omega^{(H) a b}, \Omega^{(H) I J}\right)$, satisfy the following set of Maurer-Cartan equations:

$$
\begin{aligned}
& d V^{0}=0, \\
& d V^{a I}+\frac{1}{2} \Omega_{b}^{(H) a} \wedge V^{b I}+\frac{1}{2} \Omega^{(H) I} \wedge V^{a J}=0, \\
& d V^{\alpha}-\frac{1}{8}\left(\Gamma_{a b}\right)_{\beta}{ }^{\alpha} \Omega^{(H) a b} \wedge V^{\beta}-\frac{1}{8}\left(\Gamma_{I J}\right)_{\beta}{ }^{\alpha} \Omega^{(H) I J} \wedge V^{\beta}+V^{0} \wedge V^{\alpha}=0, \\
& d V^{\bullet}+C_{\alpha \beta} V^{\alpha} \wedge V^{\beta}+2 V^{0} \wedge V^{\bullet}=0 .
\end{aligned}
$$

The metric $g_{A B}$ on the tangent space at the origin is defined as follows:

$$
g_{A B}:\left\{\begin{array}{l}
g_{\bullet \bullet}=1=g_{00} \\
g_{a I, b J}=\frac{1}{8} \delta_{a b} \delta_{I J} . \\
g_{\alpha \beta}=\delta_{\alpha \beta}
\end{array}\right.
$$

The metric connection $\omega_{A}^{B}$ satisfies the property

$$
\omega_{A}^{C} g_{C B}=-\omega_{B}^{C} g_{C A}
$$

and is the solution of the zero-torsion condition:

$$
T^{A} \equiv D V^{A}=d V^{A}+\omega_{B}^{A} \wedge V^{B}=0 .
$$

The explicit form for the spin connection is:

$$
\begin{aligned}
\omega_{B}{ }^{C} & =\tilde{\omega}_{B}{ }^{C}+\omega^{(H)}{ }_{B}{ }^{C}, \\
\omega^{(H)}{ }_{B}{ }^{C} & =\Omega^{(H) \lambda} C_{\lambda B}{ }^{C}, \\
\tilde{\omega}_{B}{ }^{C} & =\frac{1}{2}\left(C_{A B}{ }^{C}+g^{C C^{\prime}} g_{A A^{\prime}} C_{C^{\prime} B}{ }^{A^{\prime}}+g^{C C^{\prime}} g_{B B^{\prime}} C_{C^{\prime} A} B^{\prime}\right) V^{A},
\end{aligned}
$$

where $\omega^{(H)}{ }_{B} C$ is the $H$-connection which is expressed in terms of the $\Omega^{(H)} \lambda_{\text {-forms, }}$, determined by solving the second of eqs. (2.11), see eqs. (C.70). In the symmetric case the isometry group $G$ is generated by a semisimple Lie algebra $\mathfrak{g}$ which can be decomposed into its maximal compact subalgebra $\mathfrak{h} \subset \mathfrak{g}$ and the orthogonal complement $\mathfrak{t}$ to $\mathfrak{h}$ which is spanned by non-compact generators (Cartan decomposition). Choosing the basis $\left\{T_{A}\right\}$ for the tangent space of the manifold in $\mathfrak{t}$, we have $C_{A B}{ }^{C} \equiv 0$, which in turn implies $\tilde{\omega} \equiv 0$ in eqs. (2.15). Therefore in the symmetric case we can use the Cartan decomposition of the isometry algebra to define a basis of generators with respect to which the spin connection coincides with the $H$-connection: $\omega \equiv \omega^{(H)}$. This is not the case for non-symmetric manifolds, for which $\tilde{\omega}$ is always non-vanishing. The components of $\tilde{\omega}$ are given in Appendix $[$,

In terms of $\omega$ the curvature 2 -form is defined as:

$$
R_{A}{ }^{B}=d \omega_{A}^{B}-\omega_{A}^{C} \wedge \omega_{C}{ }^{B}=-\frac{1}{2} R_{C D, A}{ }^{B} V^{C} \wedge V^{D},
$$

or in components:

$$
R_{A B, C}{ }^{D}=-2 \tilde{\omega}_{[B, \mid C}^{E} \tilde{\omega}_{A], E}^{D}+C_{A B}^{\lambda} C_{\lambda, C}^{D}+C_{A B}^{E} \tilde{\omega}_{E, C}^{D} .
$$


The explicit form of the components of the Riemann tensor in the chosen rigid basis is given in eq. (C.72) of Appendix C.

Let us define on the tangent space the following complex structure:

$$
\begin{aligned}
V^{S} & =\frac{1}{\sqrt{2}}\left(V^{\bullet}-i V^{0}\right) ; \quad V^{I}=\frac{1}{\sqrt{2}}\left(V^{1 I}+i V^{0 I}\right), \\
V^{i} & =\frac{1}{\sqrt{2}}\left(V^{i_{1}}+i V^{i_{2}}\right), \quad \alpha=\left(i_{1}, i_{2}\right), i_{1}, i_{2}=1, \ldots, \frac{d}{2},
\end{aligned}
$$

where in the indices $i_{1}$ and $i_{2}$ the charge conjugation matrix reads:

$$
C_{i_{1} i_{2}}=\delta_{i_{1} i_{2}}=-C_{i_{2} i_{1}}
$$

We shall use the indices $r, s, \ldots$ to label the complex vielbein basis: $V^{r}=\left(V^{S}, V^{I}, V^{i}\right)$. In this basis the rigid metric has the following form:

$$
g_{S \bar{S}}=1 ; g_{I \bar{J}}=\frac{1}{8} \delta_{I \bar{J}} ; g_{i \bar{\jmath}}=\delta_{i \bar{\jmath}}
$$

and the curvature tensor reads:

$$
\begin{aligned}
R_{p}{ }^{q} & =R_{r \bar{s}, p}{ }^{q} V^{r} \wedge \bar{V}^{\bar{s}}, \\
R_{r \bar{s}, p}{ }^{q} & =g_{r \bar{s}} \delta_{p}^{q}+g_{\bar{s} p} \delta_{r}^{q}-C_{r p k} \bar{C}_{\bar{s}}^{k q},
\end{aligned}
$$

where the non vanishing components of the symmetric tensor $C_{r s p}$ are:

$$
C_{S I J}=\frac{1}{8} \delta_{I J} ; \quad C_{I i j}=\frac{1}{4}\left(\boldsymbol{\Gamma}_{I}\right)_{i j}
$$

and the (complex) gamma matrices $\left(\boldsymbol{\Gamma}_{I}\right)_{i j}$ are defined in (B.66). The $C_{r s p}$ is manifestly $\mathrm{SO}(q+2)$-invariant and satisfies the relation:

$$
C_{r s p} \bar{C}_{\left(\bar{r}_{1} \bar{r}_{2}\right.}^{p} \bar{C}_{\left.\bar{r}_{3} \bar{r}_{4}\right)}^{s}-\frac{4}{3} g_{r\left(\bar{r}_{1}\right.} \bar{C}_{\left.\bar{r}_{2} \bar{r}_{3} \bar{r}_{4}\right)}=E_{r \bar{r}_{1} \bar{r}_{2} \bar{r}_{3} \bar{r}_{4}}
$$

where the non-vanishing components of the tensor $E$ are:

$$
\begin{aligned}
E_{S \overline{\imath \jmath k l}} & =\frac{1}{2} \Gamma_{\overline{\imath \jmath k l}} ; E_{i \bar{I} \jmath k l}=-\frac{1}{8}\left(\boldsymbol{\Gamma}_{I}\right)_{i}{ }^{\bar{\imath}} \Gamma_{\overline{\imath \jmath k l}}, \\
\Gamma_{i j k l} & =\sum_{I=2}^{q+3}\left(\boldsymbol{\Gamma}_{I}\right)_{(i j}\left(\boldsymbol{\Gamma}_{I}\right)_{k l)}=\sum_{m=3}^{q+3}\left(\Gamma_{m}\right)_{(i j}\left(\Gamma_{m}\right)_{k l)}-\delta_{(i j} \delta_{k l)},
\end{aligned}
$$

In the symmetric case $\Gamma_{i j k l}=0$ and therefore also the tensor $E$ vanishes identically.

\section{The attractor equations and non-BPS solutions with $Z \neq 0$}

Consider the SUSY and matter central charges, the latter written in rigid indices: $Z, Z_{r}=$ $\left(Z_{S}, Z_{I}, Z_{i}\right)$. In the $N=2$ theory the matter charges $Z_{r}$ are expressed as derivatives of the SUSY central charge $Z: Z_{r}=D_{r} Z$. In terms of these charges the effective potential $V_{B H}$ has the following expression

$$
V_{B H}=|Z|^{2}+g^{r \bar{r}} Z_{r} \bar{Z}_{\bar{r}}
$$


Since the attractor point is defined by minimizing $V_{B H}$, the attractor equations are $D_{r} V_{B H}=$ 0 , which, by using the identity (A.56) of special geometry, can be recast in the form

$$
2 \bar{Z} Z_{r}+i C_{r s p} g^{s \bar{s}} g^{p \bar{p}} \bar{Z}_{\bar{s}} \bar{Z}_{\bar{p}}=0 .
$$

Equations (3.27) can then be grouped in the following way:

$$
\begin{aligned}
(a) \quad: & 2 \bar{Z} Z_{S}+8 i \sum_{I}\left(\bar{Z}_{\bar{I}}\right)^{2}=0, \\
(b)_{I} & : \quad 2 \bar{Z} Z_{I}+2 i \bar{Z}_{\bar{S}} \bar{Z}_{\bar{I}}+\frac{i}{4}\left(\boldsymbol{\Gamma}_{I}\right)_{i j} \bar{Z}_{\bar{\imath}} \bar{Z}_{\bar{\jmath}}=0, \\
(c)_{i}: & 2 \bar{Z} Z_{i}+4 i\left(\boldsymbol{\Gamma}_{I}\right)_{i j} \bar{Z}_{\bar{I}} \bar{Z}_{\bar{\jmath}}=0 .
\end{aligned}
$$

From equations $(c)_{i}$ and the property (B.67) we deduce, in the case $Z \neq 0$, the following relations:

$$
\begin{aligned}
\left(\boldsymbol{\Gamma}_{I}\right)_{i j} \bar{Z}_{\bar{I}} \bar{Z}_{\bar{\jmath}}=\frac{i}{2} \bar{Z} Z_{i}, \\
\left(\boldsymbol{\Gamma}_{I}\right)_{i j} Z_{I} \bar{Z}_{\bar{\jmath}}=\frac{2 i}{Z} Z_{i} \sum_{I}\left(Z_{I}\right)^{2} .
\end{aligned}
$$

Consider now the following combination of equations $(b)_{I}$ :

$$
\begin{aligned}
(b)_{I}\left(\boldsymbol{\Gamma}_{I}\right)_{i j} \bar{Z}_{\bar{\jmath}}: \quad 0 & =2\left(\boldsymbol{\Gamma}_{I}\right)_{i j} \bar{Z} Z_{I} \bar{Z}_{\bar{\jmath}}+2 i \bar{Z}_{\bar{S}}\left(\boldsymbol{\Gamma}_{I}\right)_{i j} \bar{Z}_{\bar{I}} \bar{Z}_{\bar{\jmath}}+\frac{i}{4}\left(\boldsymbol{\Gamma}_{I}\right)_{i j}\left(\boldsymbol{\Gamma}_{I}\right)_{k l} \bar{Z}_{\bar{\jmath}} \bar{Z}_{\bar{k}} \bar{Z}_{\bar{l}}= \\
= & 4 i \frac{\bar{Z}}{Z} Z_{i} \sum_{I}\left(Z_{I}\right)^{2}-\bar{Z}_{\bar{S}} \bar{Z} Z_{i}+\frac{i}{4} \Gamma_{i j k l} \bar{Z}_{\bar{\jmath}} \bar{Z}_{\bar{k}} \bar{Z}_{\bar{l}}
\end{aligned}
$$

where we have used the relations (3.29) and (3.30). Equation (a) can then be written in the form

$$
\sum_{I}\left(Z_{I}\right)^{2}=-\frac{i}{4} Z \bar{Z}_{\bar{S}} .
$$

Using (3.32), equation (3.31) yields

$$
\Gamma_{i j k l} \bar{Z}_{\bar{\jmath}} \bar{Z}_{\bar{k}} \bar{Z}_{\bar{l}}=0
$$

which, in virtue of eqs. (2.24), implies that:

$$
\bar{E}_{\bar{r} r_{1} r_{2} r_{3} r_{4}} \bar{Z}^{r_{1}} \bar{Z}^{r_{2}} \bar{Z}^{r_{3}} \bar{Z}^{r_{4}}=0 .
$$

Note that this result holds also in the $Z=0$ case. On the other hand, from eqs. (3.27) and (2.23) we find the relation:

$$
\bar{Z} \bar{Z}_{\bar{r}}\left(|Z|^{2}-\frac{1}{3} Z_{r} \bar{Z}_{\bar{s}} g^{r \bar{s}}\right)=-\frac{i}{8} \bar{E}_{\bar{r} r_{1} r_{2} r_{3} r_{4}} \bar{Z}^{r_{1}} \bar{Z}^{r_{2}} \bar{Z}^{r_{3}} \bar{Z}^{r_{4}}
$$

from which we deduce that for all solutions to eq. (3.27) with $Z \neq 0$ and $\bar{Z}_{\bar{r}} \neq 0$ (non-BPS) the following sum-rule holds:

$$
|Z|^{2}+Z_{r} \bar{Z}_{\bar{s}} g^{r \bar{s}}=4|Z|^{2},
$$

\footnotetext{
${ }^{1}$ This sum-rule was also found in [6] for a class of non-BPS solutions of special geometries based on generic cubic prepotentials (d-geometries).
} 
namely that the non-BPS attractors with $Z \neq 0$ feature the same value of the invariant which characterizes the corresponding orbit in the symmetric case and which is related to the entropy by eq (1.1)

$$
S=4 \pi|Z|_{\mid \text {extr. }}^{2}
$$

For BPS solutions $Z_{r} \equiv 0$ and the entropy reduces to

$$
S=\pi|Z|_{\mid e x t r}^{2}
$$

\section{The $Z=0$ non-BPS attractors}

If $Z=0$ the attractor equations in rigid indices (3.27) reduce to

$$
C_{r s p} g^{s \bar{s}} g^{p \bar{p}} \bar{Z}_{\bar{s}} \bar{Z}_{\bar{p}}=0
$$

which can also be written as follows

$$
\begin{aligned}
(a) & : \sum_{I}\left(\bar{Z}_{\bar{I}}\right)^{2}=0, \\
(b)_{I} & : \bar{Z}_{\bar{S}} \bar{Z}_{\bar{I}}+\frac{1}{8}\left(\boldsymbol{\Gamma}_{I}\right)_{i j} \bar{Z}_{\bar{\imath}} \bar{Z}_{\bar{\jmath}}=0, \\
(c)_{i}: & \left(\boldsymbol{\Gamma}_{I}\right)_{i j} \bar{Z}_{\bar{I}} \bar{Z}_{\bar{\jmath}}=0 .
\end{aligned}
$$

Recall that $Z_{i}, \bar{Z}_{\bar{\imath}}$ are in general $P+\dot{P}$ copies of the $\mathrm{SO}(q+2)$ spinorial representation and there is a further $\mathcal{S}_{q}(P, \dot{P})$ global symmetry mixing the various copies. The compact global symmetry group of the theory is therefore $\mathrm{SO}(2) \times \mathrm{SO}(2+q) \times \mathcal{S}_{q}(P, \dot{P})$.

Let us first give a general discussion of these attractors and then consider some explicit cases.

$Z_{I} \neq 0$ case. Consider first the case in which $Z_{I}$ are not all vanishing. Equation $(a)$ can be solved by taking as only non-vanishing charges $Z_{I}, Z_{2}$ and $Z_{3}$ and further requiring that $Z_{2}=-i \epsilon Z_{3}$, where $\epsilon= \pm 1$. The group $\mathrm{SO}(q+2)$ is now broken to $\mathrm{SO}(q)$. Equations $(c)_{i}$ impose then a "chirality" constraint on the spinorial charges $Z_{i}$

$$
\left(\gamma_{3}\right)_{i j} \bar{Z}_{\bar{\jmath}}=\epsilon \bar{Z}_{\bar{\imath}}
$$

while equations $(b)_{I}$ can be solved in $Z_{S}$ to give

$$
\bar{Z}_{\bar{S}}=-\frac{\epsilon}{8 \bar{Z}_{3}} \sum_{i}\left(\bar{Z}_{\bar{\imath}}\right)^{2}
$$

If the non-vanishing $Z_{i}, \bar{Z}_{\bar{\imath}}$ consist of just one copy of the spinorial representation, the $\mathrm{SO}(q)$ is further broken to the stability group of the $\mathrm{SO}(q)$ spinorial representations and $\mathcal{S}_{q}(P, \dot{P})$ is broken either to $\mathcal{S}_{q}(P-1, \dot{P})$ or to $\mathcal{S}_{q}(P, \dot{P}-1)$. If more independent copies of the spinorial representation are non-vanishing then the stability group will in general be smaller. 
$Z_{I}=0$ case. The symmetry group $\mathrm{SO}(q+2)$ is only broken by the spinorial charges to their stability subgroup. The only non trivial equations are the $(b)_{I}$ which read

$$
\left(\boldsymbol{\Gamma}_{I}\right)_{i j} \bar{Z}_{\bar{\imath}} \bar{Z}_{\bar{\jmath}}=0
$$

These equations have in general solutions if more copies of the spinorial representation are present, namely if $(P+\dot{P})>1$. For $q>1$, we can also have solutions of (4.43) if $P+\dot{P}=1$. The general solution may therefore involve one or more copies of the spinorial representation, thus breaking $\mathrm{SO}(q+2)$ to the stabilizer of the spinorial representation or to a smaller group respectively. Below we shall work out three explicit examples of non symmetric manifolds.

\subsection{The $L(0, P, \dot{P})$ space}

If $q=0$ the spinor charges $Z_{i}$ split into two sets which we shall denote by $\chi_{m}, \chi_{\dot{m}}$, where $m=1, \ldots, P$ and $\dot{m}=1, \ldots, \dot{P}$. The compact global symmetry group of the theory is $\mathrm{SO}(2) \times \mathrm{SO}(2) \times \mathrm{SO}(P) \times \mathrm{SO}(\dot{P})$. The attractor equations (4.40) read:

$$
\begin{aligned}
(a) & :\left(\bar{Z}_{\overline{2}}\right)^{2}+\left(\bar{Z}_{\overline{3}}\right)^{2}=0, \\
(b)_{2} & : \bar{Z}_{\bar{S}} \bar{Z}_{\overline{2}}+\frac{i}{8}\left(\sum_{m}\left(\bar{\chi}_{\bar{m}}\right)^{2}+\sum_{\dot{m}}\left(\bar{\chi}_{\dot{m}}\right)^{2}\right)=0, \\
(b)_{3} & : \bar{Z}_{\bar{S}} \bar{Z}_{\overline{3}}+\frac{1}{8}\left(\sum_{m}\left(\bar{\chi}_{\bar{m}}\right)^{2}-\sum_{\dot{m}}\left(\bar{\chi}_{\dot{m}}\right)^{2}\right)=0, \\
(c)_{m} & :\left(\bar{Z}_{\overline{2}}-i \bar{Z}_{\overline{3}}\right) \bar{\chi}_{\bar{m}}=0, \\
(c)_{\dot{m}} & : \quad\left(\bar{Z}_{\overline{2}}+i \bar{Z}_{\overline{3}}\right) \bar{\chi}_{\dot{m}}=0 .
\end{aligned}
$$

Let us consider the various orbits of solutions.

The $Z_{S} \neq 0, Z_{I} \neq 0$ case. If $\bar{Z}_{\overline{2}}=i \bar{Z}_{\overline{3}} \neq 0$ equation $(a)$ and equations $(c)_{m}$ are satisfied while equations $(c)_{\dot{m}}$ imply $\chi_{\dot{m}}=0$. Equations $(b)_{I}$ imply

$$
\bar{Z}_{\bar{S}}=-\frac{i}{8 \bar{Z}_{\overline{2}}} \sum_{m}\left(\bar{\chi}_{\bar{m}}\right)^{2} .
$$

These solutions define an orbit with stability group $\mathrm{SO}(P-1) \times \mathrm{SO}(\dot{P})$.

The $Z_{S}=0, Z_{I} \neq 0$ case. If $\bar{Z}_{\overline{2}}=i \bar{Z}_{\overline{3}} \neq 0$ we still have $\chi_{\dot{m}}=0$ while from equations $(b)_{I}$ we have $\sum_{m}\left(\bar{\chi}_{\bar{m}}\right)^{2}=0$, which may have a trivial solution $\bar{\chi}_{\bar{m}}=0$ with stability group $\mathrm{SO}(P) \times \mathrm{SO}(\dot{P})$ and a non-trivial solution

$$
\bar{\chi}_{\overline{1}}= \pm i \bar{\chi}_{\overline{2}} \neq 0 \quad \bar{\chi}_{\bar{m} \neq 1,2}=0
$$

with stability group $\mathrm{SO}(P-2) \times \mathrm{SO}(\dot{P})$. 
The $Z_{S} \neq 0, Z_{I}=0$ case. Equations $(b)_{I}$ imply

$$
\sum_{m}\left(\bar{\chi}_{\bar{m}}\right)^{2}=\sum_{\dot{m}}\left(\bar{\chi}_{\dot{m}}\right)^{2}=0
$$

which can have the following solutions with the corresponding stability groups

$$
\begin{aligned}
& \bar{\chi}_{\bar{m}}=\bar{\chi}_{\dot{m}}=0 \quad \text { stability group: } \mathrm{SO}(2) \times \mathrm{SO}(P) \times \mathrm{SO}(\dot{P}), \\
& \left.\begin{array}{c}
\bar{\chi}_{\overline{1}}= \pm i \bar{\chi}_{\overline{2}} \neq 0 \\
\bar{\chi}_{\bar{m} \neq 1,2}=0=\bar{\chi}_{\dot{m}}
\end{array}\right\} \quad \text { stability group: } \mathrm{SO}(P-2) \times \mathrm{SO}(\dot{P}), \\
& \left.\begin{array}{cc}
\bar{\chi}_{\overline{1}}= \pm i \bar{\chi}_{\overline{2}} \neq 0 & \bar{\chi}_{\bar{m} \neq 1,2}=0 \\
\bar{\chi}_{\dot{\overline{1}}}= \pm i \bar{\chi}_{\overline{\overline{2}}} & \bar{\chi}_{\dot{\bar{m}} \neq 1,2}=0
\end{array}\right\} \quad \text { stability group: } \operatorname{SO}(P-2) \times \operatorname{SO}(\dot{P}-2),
\end{aligned}
$$

\subsection{The $L(1,2)$ space}

The global symmetry group in this case is $\mathrm{SO}(2) \times \mathrm{SO}(3) \times \mathrm{SO}(2), \mathrm{SO}(2)$ being $\mathcal{S}_{1}(2)$ for this manifold. Here we shall denote by $\vec{\chi}_{m} \equiv\left(\chi_{\alpha, m}\right)$ the spinorial central charges $Z_{i} \equiv Z_{\alpha, m}$, where $\alpha=1,2$ is the spinorial index while $m=1,2$ runs over the number of copies. The vector charges are $Z_{I}=\left(Z_{2}, Z_{3}, Z_{4}\right)$. We can choose the relevant gamma matrices to have the form:

$$
\boldsymbol{\Gamma}_{2}=i \mathbb{1}_{2} \times \mathbb{1}_{2} ; \boldsymbol{\Gamma}_{3}=\sigma_{3} \times \mathbb{1}_{2} ; \boldsymbol{\Gamma}_{4}=\sigma_{1} \times \mathbb{1}_{2}
$$

If we choose $Z_{4}=0$ and $Z_{2}=-i \epsilon Z_{3} \neq 0$ equation (4.41) has the following solutions

$$
\begin{array}{lll}
\epsilon=+1: & \vec{\chi}_{m}=\left(\chi_{m}, 0\right), \\
\epsilon=-1 \quad: & \vec{\chi}_{m}=\left(0, \chi_{m}\right) .
\end{array}
$$

A non-vanishing spinor breaks $\mathrm{SO}(3) \times \mathrm{SO}(2)$ completely. Equation (4.42) reads

$$
\bar{Z}_{\bar{S}}=-\frac{\epsilon}{8 \bar{Z}_{3}} \sum_{\bar{m}=1}^{2}\left(\bar{\chi}_{\bar{m}}\right)^{2} .
$$

In the case $\bar{Z}_{\bar{S}}=0$ we need to have either $\chi_{m}=0$ or $\chi_{1}= \pm i \chi_{2} \neq 0$. In the first case the group $\mathrm{SO}(2)$ acting on the $m$ index is restored.

If $Z_{I}=0$ a solution of (4.43) must involve both copies $\vec{\chi}_{m}$ and moreover $\vec{\chi}_{1}= \pm i \vec{\chi}_{2}$.

\subsection{The $L(2,2)$ space}

The global symmetry group in this case is $\mathrm{SO}(2) \times \mathrm{SO}(4) \times \mathrm{U}(2)$. the spinorial charges $Z_{i}$ will be denoted as usual by $\vec{\chi}_{m} \equiv\left(\chi_{\alpha, m}\right)$, where $\alpha=1, \ldots, 4$ is the spinorial index and $m=1,2$. The gamma matrices can be chosen of the form

$$
\begin{aligned}
& \boldsymbol{\Gamma}_{2}=i \mathbb{1}_{2} \times \mathbb{1}_{2} \times \mathbb{1}_{2} ; \boldsymbol{\Gamma}_{3}=\sigma_{1} \times \sigma_{1} \times \mathbb{1}_{2} ; \boldsymbol{\Gamma}_{4}=\sigma_{3} \times \sigma_{1} \times \mathbb{1}_{2}, \\
& \boldsymbol{\Gamma}_{5}=\mathbb{1}_{2} \times \sigma_{3} \times \mathbb{1}_{2} .
\end{aligned}
$$


If $Z_{2}=-i \epsilon Z_{3} \neq 0$ and $Z_{4}=Z_{5}=0, \mathrm{SO}(4)$ is broken to $\mathrm{SO}(2)$. In this case the solution to (4.41) has the form

$$
\vec{\chi}_{m}=\left(\epsilon \chi_{1, m}, \epsilon \chi_{2, m}, \chi_{2, m}, \chi_{1, m}\right) .
$$

If just one copy of the spinorial charge is non-vanishing, $\mathrm{U}(2)$ is broken to $\mathrm{U}(1)$. Equation (4.42) then reads

$$
\bar{Z}_{\bar{S}}=-\frac{\epsilon}{4 \bar{Z}_{3}} \sum_{\bar{m}=1}^{2}\left[\left(\bar{\chi}_{1, \bar{m}}\right)^{2}+\left(\bar{\chi}_{2, \bar{m}}\right)^{2}\right] .
$$

If $Z_{S}=0$ the above equation can be satisfied in three possible ways: All vanishing spinorial charges, in which case the residual group is $\mathrm{SO}(2) \times \mathrm{U}(2)$; Just one non vanishing spinorial charge, in which case the residual group is $\mathrm{U}(1)$; Two independent copies of spinorial charges in which case there is no residual symmetry.

In the $Z_{I}=0$ case, the $\mathrm{SO}(4)$ is broken by the spinorial charges only. Equation (4.43), as opposed to the $q=1$ case, can have non-vanishing solutions with just one copy of the spinorial charges, in which case $\mathrm{U}(2)$ is broken to $\mathrm{U}(1)$. The possible solutions are

$$
\begin{aligned}
\vec{\chi}_{m} & =\left(\chi_{1, m}, \pm i \chi_{1, m}, 0,0\right), \\
\vec{\chi}_{m} & =\left(0,0, \chi_{3, m}, \pm i \chi_{3, m}\right), \\
\vec{\chi}_{m} & =\left(\chi_{1, m}, \pm i \chi_{1, m}, \chi_{3, m}, \mp i \chi_{3, m}\right), \\
\vec{\chi}_{1} & = \pm i \vec{\chi}_{2} .
\end{aligned}
$$

If just one copy of the spinorial charges is non vanishing $\mathrm{SO}(4)$ is broken to $\mathrm{SO}(3)_{\text {diag }}$.

\section{Conclusions}

In the present paper we have extended the analysis of the attractor equations to $N=2$ models featuring a generic homogeneous space. In particular we have found that no deviation with respect to the symmetric case occurs in the $Z \neq 0$ analysis of non-BPS solutions, namely the Bertotti-Robinson mass $M_{B R}=2|Z|_{\text {extr. }}$. We leave for future work the study of the mass spectra at the critical points and a detailed analysis of higher curvature corrections which encode possible deviations from Einstein supergravity.

\section{Acknowledgements}

Work supported in part by the European Community's Human Potential Program under contract MRTN-CT-2004-005104 'Constituents, fundamental forces and symmetries of the universe', in which R. D'A. and M.T. are associated to Torino University. The work of S.F. has been supported in part by European Community's Human Potential Program under contract MRTN-CT-2004-005104 'Constituents, fundamental forces and symmetries of the universe' and the contract MRTN- CT-2004-503369 'The quest for unification: Theory Confronts Experiments', in association with INFN Frascati National Laboratories and by D.O.E. grant DE-FG03-91ER40662, Task C. 


\section{A Some useful properties of special Kähler manifolds}

A special Kähler manifold $\mathcal{M}$ of complex dimension $n$ is a Kähler-Hodge manifold which can be characterized in the following way (see for example [25] and references therein). Let us introduce a $(2 n+2)$-dimensional section $V(z, \bar{z})$ of a symplectic $\mathrm{U}(1)$ bundle over $\mathcal{M}$. The manifold is special Kähler if $V$ satisfies the following differential conditions

$$
\begin{aligned}
D_{r} D_{s} V & =i C_{r s p} g^{p \bar{p}} \bar{D}_{\bar{p}} \bar{V} \\
D_{r} D_{\bar{s}} \bar{V} & =g_{r \bar{s}} \bar{V} \\
D_{s} \bar{V} & =0
\end{aligned}
$$

where the indices $r, s, \ldots=1, \ldots, n$ are flat indices and the covariant derivative $D_{r}$ contains the $\mathrm{U}(1)$ and Levi-Civita connections. In particular integrability of the above identities require the Riemann tensor to have the following form

$$
\begin{aligned}
R_{p}^{q} & =R_{r \bar{s}, p^{q}} V^{r} \wedge \bar{V}^{\bar{s}}, \\
R_{r \bar{s}, p}{ }^{q} & =g_{r \bar{s}} \delta_{p}^{q}+g_{\bar{s} p} \delta_{r}^{q}-C_{r p k} \bar{C}_{\bar{s}}^{k p} .
\end{aligned}
$$

If we write

$$
V=\left(\begin{array}{c}
L^{\Lambda} \\
M_{\Sigma}
\end{array}\right) ; \Lambda, \Sigma=0, \ldots, n
$$

the SUSY central charge $Z$ is defined as follows in terms of $V$ and of the quantized electric and magnetic charges $q_{\Lambda}, p^{\Lambda}$ as follows

$$
Z=M_{\Lambda} p^{\Lambda}-L^{\Lambda} q_{\Lambda}
$$

while the matter central charges $Z_{r}$, in rigid indices, are given by $Z_{r}=D_{r} Z$.

\section{B Clifford module}

We use the following $2 d$-dimensional (reducible) representation for the $\mathrm{SO}(2, q+2)$ Clifford algebra:

$$
\begin{aligned}
& \left\{\mathbb{\Gamma}_{\Lambda}, \mathbb{\Gamma}_{\Sigma}\right\}=2 \eta_{\Lambda \Sigma} ; \eta_{\Lambda \Sigma}=\operatorname{diag}(-1,-1,+1, \ldots,+1), \\
& \mathbb{\Gamma}_{\Lambda}=\left(\begin{array}{cc}
\mathbf{0}_{d} & \left(\Gamma_{\Lambda}\right)_{\alpha \dot{\alpha}} \\
\left(\Gamma_{\Lambda}\right)^{\dot{\alpha} \alpha} & \mathbf{0}_{d}
\end{array}\right) \\
& \Lambda, \Sigma=0, \ldots, q+3 ; \alpha, \dot{\alpha}=1, \ldots, d \text {. }
\end{aligned}
$$

The explicit matrix form for $\mathbb{\Gamma}_{\Lambda}$ that we shall use is, for $q>0$, the following:

$$
\begin{aligned}
\mathbb{\Gamma}_{m} & =\gamma_{m} \times \sigma_{2} \times \sigma_{2} ; m=3, \ldots, q+3, \\
\mathbb{\Gamma}_{2} & =\mathbb{1}_{\frac{d}{2}} \times \mathbb{1}_{2} \times \sigma_{1}, \\
\mathbb{\Gamma}_{1} & =\mathbb{1}_{\frac{d}{2}} \times i \sigma_{3} \times \sigma_{2}, \\
\mathbb{\Gamma}_{0} & =\mathbb{1}_{\frac{d}{2}} \times i \sigma_{1} \times \sigma_{2},
\end{aligned}
$$


where the $\frac{d}{2} \times \frac{d}{2}$ matrices $\gamma_{m}$ generate the $\mathrm{SO}(q+1)$-Clifford algebra. The charge conjugation matrix is:

$$
C^{(-)}=\mathbb{1}_{\frac{d}{2}} \times i \sigma_{2} \times \sigma_{3}=\left(\begin{array}{cc}
C_{\alpha \beta} & \mathbf{0}_{d} \\
\mathbf{0}_{d} & C^{\dot{\alpha} \dot{\beta}}
\end{array}\right) ; \quad C^{T}=-C ; C^{2}=-\mathbb{1}_{d} .
$$

We also define the (complex) $\mathrm{SO}(q+2)$ gamma matrices

$$
\hat{\boldsymbol{\Gamma}}_{I}=\left(\begin{array}{cc}
\mathbf{0} & \boldsymbol{\Gamma}_{I} \\
\overline{\boldsymbol{\Gamma}}_{I} & \mathbf{0}
\end{array}\right)
$$

where the off-diagonal blocks $\boldsymbol{\Gamma}_{I}, \overline{\boldsymbol{\Gamma}}_{I}$ are defined as follows:

$$
\begin{aligned}
\left(\boldsymbol{\Gamma}_{2}\right)_{i j} & =i \delta_{i j} ; \quad\left(\boldsymbol{\Gamma}_{m}\right)_{i j}=\left(\gamma_{m}\right)_{i j} \\
\overline{\boldsymbol{\Gamma}}_{I} & =\left(\boldsymbol{\Gamma}_{I}\right)^{*}
\end{aligned}
$$

and satisfy the relation:

$$
\left\{\overline{\boldsymbol{\Gamma}}_{I}, \boldsymbol{\Gamma}_{J}\right\}=2 \delta_{I J}
$$

\section{Spin connection and the curvature tensor in components}

From the Maurer-Cartan equations (2.11) we may deduce the structure constants:

$$
\begin{aligned}
C_{a b, c I}{ }^{d J} & =\delta_{I}^{J} \delta_{[a}^{d} \eta_{b] c}=-\delta_{I}^{J} \delta_{[a}^{d} \delta_{b] c}, \\
C_{I J, a K}{ }^{b L} & =\delta_{a}^{b} \delta_{[I}^{L} \eta_{J] K}=\delta_{a}^{b} \delta_{[I}^{L} \delta_{J] K}, \\
C_{a I, b J}{ }^{c d} & =-\delta_{a b}^{c d} \eta_{I J}=-\delta_{a b}^{c d} \delta_{I J}, \\
C_{a I, b J}{ }^{K L} & =-\delta_{I J}^{K L} \eta_{a b}=\delta_{I J}^{K L} \delta_{a b}, \\
C_{\Lambda \Sigma, \alpha}{ }^{\beta} & =-\frac{1}{4}\left(\Gamma_{\Lambda \Sigma}\right)_{\alpha}{ }^{\beta}, \\
C_{\alpha \beta} & =2 C_{\alpha \beta} ; C_{0 \alpha}{ }^{\beta}=\delta_{\alpha}^{\beta} ; C_{0} \bullet=2 .
\end{aligned}
$$

The non-zero constant components $\tilde{\omega}_{A, B}{ }^{C}$ of the spin connection are:

$$
\begin{aligned}
\tilde{\omega}_{\bullet, \alpha}{ }^{\beta} & =\frac{1}{2} C_{\beta^{\prime} \alpha} \bullet g^{\beta \beta^{\prime}} g_{\bullet \bullet}=-C_{\alpha \beta}, \\
\tilde{\omega}_{\alpha, \bullet}{ }^{\beta} & =\frac{1}{2} C_{\beta^{\prime} \alpha} \bullet g^{\beta \beta^{\prime}} g_{\bullet \bullet}=-C_{\alpha \beta}, \\
\tilde{\omega}_{\alpha \beta} \bullet & =C_{\alpha \beta}, \\
\tilde{\omega}_{a b, \alpha}{ }^{\beta} & =-\frac{1}{4}\left(\Gamma_{a b}\right)_{\alpha}^{\beta} ; \tilde{\omega}_{I J, \alpha}{ }^{\beta}=-\frac{1}{4}\left(\Gamma_{I J}\right)_{\alpha}{ }^{\beta}, \\
\tilde{\omega}_{\bullet, 0} \bullet & =-2 ; \tilde{\omega}_{\bullet, \bullet}{ }^{0}=2 g^{00} g_{\bullet \bullet}=2, \\
\tilde{\omega}_{\alpha, 0}{ }^{\beta} & =-\delta_{\alpha}^{\beta} ; \tilde{\omega}_{\alpha, \beta}=g_{\beta \alpha} g^{00}=\delta_{\alpha \beta}, \\
\tilde{\omega}_{\alpha, a I} \beta & =\frac{1}{4}\left(\Gamma_{a I}\right)_{\alpha}{ }^{\beta} ; \tilde{\omega}_{\alpha, \beta}{ }^{a I}=-\frac{1}{4}\left(\Gamma_{a^{\prime} I^{\prime}}\right)_{\alpha}{ }^{\beta^{\prime}} g^{a^{\prime} I^{\prime} a I} g_{\beta \beta^{\prime}}=-2\left(\Gamma_{a I}\right)_{\alpha}{ }^{\beta} .
\end{aligned}
$$

For completeness, let us give the explicit form of the components of the $H$-connection $\Omega^{(H)}$ in (2.15), obtained by solving the second of eqs. (2.11)

$$
\begin{aligned}
\Omega^{(H)}{ }_{a I, b}^{c},_{b} & =\left(\frac{2}{q+2}\right)\left(V_{b J}^{u} V_{a I}^{v} \partial_{[u} V_{v]}^{c J}+V_{a I}^{u} V^{c J v} \partial_{[u} V_{v] b J}-V^{c J u} V_{b J}^{v} \partial_{[u} V_{v] a I}\right), \\
\Omega^{(H)}{ }_{a I,}{ }^{K}{ }_{J} & =V_{b J}^{u} V_{a I}^{v} \partial_{[u} V_{v]}^{b K}+V_{a I}^{u} V^{b K v} \partial_{[u} V_{v] b J}-V^{b K u} V_{b J}{ }^{v} \partial_{[u} V_{v] a I},
\end{aligned}
$$


$u, v=1, \ldots, 2 n$ are curved indices on the scalar manifold and $V_{a I}{ }^{u}$ denotes the inverse vielbein:

$$
V_{a I}^{u} V_{u}^{b J}=\delta_{a}^{b} \delta_{I}^{J}
$$

From eq. (2.17) we deduce the components of the Riemann tensor:

$$
\begin{aligned}
& R_{a I b J, c K}^{d L}=-\frac{1}{2} \delta_{[a}^{d} \eta_{b] c} \eta_{I J} \delta_{L}^{K}-\frac{1}{2} \delta_{[I}^{L} \eta_{J] K} \eta_{a b} \delta_{c}^{d}= \\
& =\frac{1}{2} \delta_{[a}^{d} \delta_{b] c} \delta_{I J} \delta_{L}^{K}+\frac{1}{2} \delta_{[I}^{L} \delta_{J] K} \delta_{a b} \delta_{c}^{d} \\
& R_{\alpha \beta, a I}^{b J}=\frac{1}{8}\left(\Gamma_{a b^{\prime}}\right)_{\alpha}^{\beta^{\prime}} \eta_{I J^{\prime}} g^{b^{\prime} J^{\prime}} g_{\beta \beta^{\prime}}+\frac{1}{8}\left(\Gamma_{I J^{\prime}}\right)_{\alpha}^{\beta^{\prime}} \eta_{a b^{\prime}} g^{b^{\prime} J^{\prime}} g_{\beta \beta^{\prime}}= \\
& =\left(\Gamma_{a b}\right)_{\alpha}^{\beta} \delta_{I J}-\left(\Gamma_{I J}\right)_{\alpha}^{\beta} \delta_{a b}, \\
& R_{a I \alpha, b J^{\beta}}=-\frac{1}{16}\left(\Gamma_{a I}\right)_{\alpha}^{\gamma}\left(\Gamma_{b J}\right)_{\gamma}^{\beta} \text {, } \\
& R_{\alpha \beta, 0}{ }^{\bullet}=-2 C_{\alpha \beta}, \\
& R_{\alpha \bullet a I}{ }^{\beta}=-\frac{1}{4}\left(\Gamma_{a I}\right)_{\alpha}^{\gamma} C_{\gamma \beta^{\prime}} g^{\beta^{\prime} \beta} g_{\bullet \bullet}=-\frac{1}{4}\left(\Gamma_{a I}\right)_{\alpha}^{\gamma} C_{\gamma \beta}, \\
& R_{\alpha \bullet, 0}{ }^{\beta}=-C_{\alpha \beta^{\prime}} g^{\beta^{\prime} \beta}=-C_{\alpha \beta}, \\
& R_{a I \alpha, 0}{ }^{\beta}=\frac{1}{4}\left(\Gamma_{a I}\right)_{\alpha}^{\beta}, \\
& R_{0 \alpha, a I^{\beta}}=\frac{1}{4}\left(\Gamma_{a I}\right)_{\alpha}^{\beta}, \\
& R_{0 \alpha, 0}{ }^{\beta}=-\delta_{\alpha}^{\beta} \text {, } \\
& R_{0} \bullet 0^{\bullet}=-4 \text {, } \\
& R_{a I b J, \alpha}{ }^{\beta}=\frac{1}{8}\left(\Gamma_{a b}\right)_{\alpha}{ }^{\beta} \delta_{I J}-\frac{1}{8}\left(\Gamma_{I J}\right)_{\alpha}{ }^{\beta} \delta_{a b}, \\
& R_{a I \alpha, \beta} \cdot=-\frac{1}{4}\left(\Gamma_{a I}\right)_{\alpha}^{\gamma} C_{\gamma \beta}, \\
& R_{0} \bullet \alpha^{\beta}=-2 C_{\alpha \gamma} g^{\gamma \beta}=-2 C_{\alpha \beta}, \\
& R_{0 \alpha, \beta} \cdot=C_{\alpha \beta} \text {, } \\
& R_{\alpha \beta, \gamma}{ }^{\delta}=-2 C_{\gamma \delta^{\prime}} C_{\alpha \beta} g^{\delta^{\prime} \delta}+2 C_{\gamma[\alpha} C_{\beta] \delta^{\prime}} g^{\delta^{\prime} \delta}-2 g_{\gamma[\alpha} g_{\beta] \delta^{\prime}} g^{\delta^{\prime} \delta}-\frac{1}{8}\left(\Gamma_{a^{\prime} I^{\prime}}\right)_{[\alpha}^{\gamma^{\prime}}\left(\Gamma_{a I}\right)_{\beta]}{ }^{\delta} g_{\gamma^{\prime} \gamma} g^{a^{\prime} I^{\prime}, a I}= \\
& =-2 C_{\gamma \delta} C_{\alpha \beta}+2 C_{\gamma[\alpha} C_{\beta] \delta}-2 g_{\gamma[\alpha} g_{\beta] \delta}-\left(\Gamma_{a I}\right)_{[\alpha}^{\gamma}\left(\Gamma_{a I}\right)_{\beta]}^{\delta} \\
& R_{\alpha \bullet \beta} \bullet=-g_{\alpha \beta}=-\delta_{\alpha \beta} \text {. }
\end{aligned}
$$

\section{The $\mathrm{SO}(1, q+1)$-covariant rigid basis}

With the exception of the minimal coupling case $L(-2, P)=\mathrm{U}(1,1+P) / \mathrm{U}(1) \times \mathrm{U}(1+P)$, all the other homogeneous special Kähler manifolds have a five dimensional "parent" manifold which is a real special manifold. This manifold always admits an $\mathrm{SO}(1,1+q)$ group of isometries, which, in most of the cases, can be extended to global symmetry group of the whole theory. We may keep track of the five dimensional origin of a special Kähler manifold, by choosing a basis for the tangent space of the four dimensional manifold which is $\mathrm{SO}(1,1+q)-$ 
covariant. To this end we decompose the $\mathfrak{s o}(2,2+q)$ algebra as follows

$$
\mathfrak{s o}(2,2+q)=\mathfrak{o}(1,1)_{1} \oplus \mathfrak{s o}(1,1+q) \oplus(\mathbf{2}+\mathbf{q})_{\frac{1}{2}} \oplus(\mathbf{2}+\mathbf{q})_{-\frac{1}{2}}
$$

where the subscripts refer to the $\mathfrak{o}(1,1)_{1}$-grading, and choose as a basis for the tangent space of the $\mathrm{SO}(2,2+q) / \mathrm{SO}(2) \times \mathrm{SO}(2+q)$ sub-manifold the following isometry generators

$$
\begin{aligned}
T_{I} & =\left\{T_{12}, T_{0 m}\right\} ; I=2, \ldots, q+3 ; m=3, \ldots, q+3, \\
T_{+I} & =\left\{T_{10}+T_{20}, T_{1 m}+T_{2 m}\right\},
\end{aligned}
$$

where $T_{12}$ is the $\mathfrak{o}(1,1)_{1}$ generator and is parametrized by the radial modulus of the internal circle in the reduction from five to four dimensions, $T_{1 m}$ generate the coset $\mathrm{SO}(1,1+q) / \mathrm{SO}(1+$ $q)$ and $T_{+I}$ are the generators of the $(q+2)$-dimensional abelian subalgebra (translations) $(\mathbf{2}+\mathbf{q})_{\frac{1}{2}}$. The new basis $\left\{T_{A}^{\prime}\right\}$ for the tangent space of the total manifold is now

$$
\left\{T_{A}^{\prime}\right\}=\left\{h_{0}, T_{I}, T_{+I}, T_{\alpha}, T_{\bullet}\right\}
$$

and we shall denote by $\left\{V^{A}\right\}=\left\{V^{0}, V^{I}, V^{+I}, V^{\alpha}, V^{\bullet}\right\}$ the dual vielbein basis. We then define the complex vielbein as follows

$$
\begin{aligned}
V^{S} & =\frac{1}{\sqrt{2}}\left(V^{\bullet}-i V^{0}\right) ; \quad V^{I}=\frac{1}{\sqrt{2}}\left(V^{+I}+i V^{I}\right), \\
V^{i} & =\frac{1}{\sqrt{2}}\left(V^{i_{1}}+i V^{i_{2}}\right), \quad \alpha=\left(i_{1}, i_{2}\right), i_{1}, i_{2}=1, \ldots, \frac{d}{2},
\end{aligned}
$$

namely the abelian subalgebra $(\mathbf{2}+\mathbf{q})_{\frac{1}{2}}$ is parametrized by the real parts of the complex scalars which span the submanifold $\mathrm{SO}(2,2+q) / \mathrm{SO}(2) \times \mathrm{SO}(2+q)$. If we write the curvature in this new basis we find the following non-vanishing components

$$
R_{r \bar{s}, p}^{q}=g_{r \bar{s}} \delta_{p}^{q}+g_{\bar{s} p} \delta_{r}^{q}-C_{r p k}^{\prime} \bar{C}_{\bar{s}}^{\prime k q}
$$

where the new symmetric tensor $C_{r s p}^{\prime}$ has the form

$$
C_{S I J}^{\prime}=\frac{1}{8} \eta_{I J} ; \quad C_{I i j}^{\prime}=\frac{1}{4}\left(\boldsymbol{\Gamma}_{I}^{\prime}\right)_{i j}
$$

$\eta_{I J}=\operatorname{diag}(-1,+1, \ldots,+1)$ being the $\mathrm{SO}(1,1+q)$-invariant tensor of the $\mathbf{q}+\mathbf{2}$ representation and $\Gamma_{I}^{\prime}, \overline{\boldsymbol{\Gamma}}_{I}^{\prime}$, defined as

$$
\begin{aligned}
& \left(\boldsymbol{\Gamma}_{2}^{\prime}\right)_{i j}=-\delta_{i j} ;\left(\boldsymbol{\Gamma}_{m}^{\prime}\right)_{i j}=\left(\gamma_{m}\right)_{i j} \\
& \left(\overline{\boldsymbol{\Gamma}}_{2}^{\prime}\right)_{i j}=\delta_{i j} ;\left(\overline{\boldsymbol{\Gamma}}_{m}^{\prime}\right)_{i j}=\left(\gamma_{m}\right)_{i j}
\end{aligned}
$$

are blocks of $\mathrm{SO}(1,1+q)$ gamma-matrices

$$
\hat{\boldsymbol{\Gamma}}_{I}^{\prime}=\left(\begin{array}{cc}
\mathbf{0} & \boldsymbol{\Gamma}_{I}^{\prime} \\
\overline{\boldsymbol{\Gamma}}_{I}^{\prime} & \mathbf{0}
\end{array}\right) \quad ; \quad\left\{\hat{\boldsymbol{\Gamma}}_{I}^{\prime}, \hat{\boldsymbol{\Gamma}}_{J}^{\prime}\right\}=2 \eta_{I J} .
$$

Note that the curvature and the $C^{\prime}$ tensors are now $\mathrm{SO}(1,1+q)$-invariant and that the change from the $\mathrm{SO}(2+q)$-covariant vielbein basis to the $\mathrm{SO}(1,1+q)$-covariant one amounts to the action of the following $\mathrm{U}(n)$ transformation:

$$
V^{I=2} \rightarrow i V^{I=2}, V^{I \neq 2} \rightarrow V^{I \neq 2} \quad, \quad V^{A \neq I} \rightarrow V^{A \neq I} .
$$




\section{References}

[1] S. Ferrara, R. Kallosh and A. Strominger, "N=2 extremal black holes," Phys. Rev. D 52, 5412 (1995) arXiv:hep-th/9508072]; A. Strominger, "Macroscopic Entropy of $N=2$ Extremal Black Holes," Phys. Lett. B 383 (1996) 39 arXiv:hep-th/9602111; S. Ferrara and R. Kallosh, "Supersymmetry and Attractors," Phys. Rev. D 54, 1514 (1996) arXiv:hep-th/9602136; S. Ferrara and R. Kallosh, "Universality of Supersymmetric Attractors," Phys. Rev. D 54 (1996) 1525 arXiv:hep-th/9603090.

[2] S. Ferrara, G. W. Gibbons and R. Kallosh, "Black holes and critical points in moduli space," Nucl. Phys. B 500, 75 (1997) arXiv:hep-th/9702103].

[3] S. Ferrara and R. Kallosh, "On N = 8 attractors," Phys. Rev. D 73, 125005 (2006) arXiv:hep-th/0603247.

[4] K. Goldstein, N. Iizuka, R. P. Jena and S. P. Trivedi, "Non-supersymmetric attractors," Phys. Rev. D 72 (2005) 124021 arXiv:hep-th/0507096.

[5] R. Kallosh, "New attractors," JHEP 0512 (2005) 022 arXiv:hep-th/0510024.

[6] P. K. Tripathy and S. P. Trivedi, "Non-supersymmetric attractors in string theory," JHEP 0603 (2006) 022 arXiv:hep-th/0511117.

[7] A. Giryavets, "New attractors and area codes," JHEP 0603 (2006) 020 arXiv:hep-th/0511215.

[8] R. Kallosh, N. Sivanandam and M. Soroush, "The non-BPS black-hole attractor equation," JHEP 0603 (2006) 060 arXiv:hep-th/0602005.

[9] G. Lopes Cardoso, B. de Wit and T. Mohaupt, "Corrections to macroscopic supersymmetric black-hole entropy," Phys. Lett. B 451 (1999) 309 arXiv:hep-th/9812082; G. Lopes Cardoso, B. de Wit, J. Kappeli and T. Mohaupt, "Stationary BPS solutions in $\mathrm{N}=2$ supergravity with $\mathrm{R}^{* *} 2$ interactions," JHEP 0012 (2000) 019 arXiv:hep-th/0009234; G. Lopes Cardoso, B. de Wit, J. Kappeli and T. Mohaupt, "Black hole partition functions and duality," JHEP 0603 (2006) 074 arXiv:hep-th/0601108.

[10] A. Strominger and C. Vafa, "Microscopic Origin of the Bekenstein-Hawking Entropy," Phys. Lett. B 379, 99 (1996) arXiv:hep-th/9601029.

[11] J. M. Maldacena, A. Strominger and E. Witten, "Black hole entropy in M-theory," JHEP 9712, 002 (1997) arXiv:hep-th/9711053.

[12] H. Ooguri, A. Strominger and C. Vafa, "Black hole attractors and the topological string," Phys. Rev. D 70 (2004) 106007 arXiv:hep-th/0405146. 
[13] A. Dabholkar, "Exact counting of black hole microstates," Phys. Rev. Lett. 94, 241301 (2005) arXiv:hep-th/0409148.

[14] A. Dabholkar, F. Denef, G. W. Moore and B. Pioline, "Precision counting of small black holes," JHEP 0510, 096 (2005) arXiv:hep-th/0507014.

[15] A. Sen, "Black hole entropy function and the attractor mechanism in higher derivative gravity," JHEP 0509, 038 (2005) arXiv:hep-th/0506177.

[16] A. Dabholkar, A. Sen and S. Trivedi, "Black hole microstates and attractor without supersymmetry," arXiv:hep-th/0611143;

[17] B. Pioline, "Lectures on on black holes, topological strings and quantum attractors," Class. Quant. Grav. 23 (2006) S981 arXiv:hep-th/0607227].

[18] S. Bellucci, S. Ferrara, M. Gunaydin and A. Marrani, "Charge orbits of symmetric special geometries and attractors," Int. J. Mod. Phys. A 21, 5043 (2006) arXiv:hep-th/0606209.

[19] D. V. Alekseevsky, "Classification of quaternionic spaces with a transitive solvable group of motions", Math. USSR Izvestija 9 (1975) 297-339.

[20] B. de Wit, F. Vanderseypen and A. Van Proeyen, "Symmetry structure of special geometries", Nucl. Phys. B400 (1993) 463-524, hep-th/9210068;

[21] V. Cortés, "Alekseevskian spaces", Diff. Geom. Appl. 6 (1996) 129-168.

[22] P. K. Tripathy and S. P. Trivedi, "Compactification with flux on K3 and tori," JHEP 0303 (2003) 028 arXiv:hep-th/0301139.

[23] L. Andrianopoli, R. D'Auria, S. Ferrara and M. A. Lledo, "4-D gauged supergravity analysis of type IIB vacua on K3 x T**2/Z(2)," JHEP 0303 (2003) 044 arXiv:hep-th/0302174].

[24] C. Angelantonj, R. D'Auria, S. Ferrara and M. Trigiante, "K3 x T**2/Z(2) orientifolds with fluxes, open string moduli and critical points," Phys. Lett. B 583 (2004) 331 arXiv:hep-th/0312019.

[25] L. Andrianopoli, M. Bertolini, A. Ceresole, R. D'Auria, S. Ferrara, P. Fre and T. Magri, " $\mathrm{N}=2$ supergravity and $\mathrm{N}=2$ super Yang-Mills theory on general scalar manifolds: Symplectic covariance, gaugings and the momentum map," J. Geom. Phys. 23 (1997) 111 arXiv:hep-th/9605032. 